\title{
Quantum kinetic description of Coulomb effects in one-dimensional nanoscale transistors
}

\author{
K. M. Indlekofer* and J. Knoch \\ Institute for Thin Films and Interfaces (ISG-1) and Center of Nanoelectronic Systems for Information Technology (CNI), Research Centre \\ Jülich GmbH, D-52425 Jülich, Germany \\ J. Appenzeller \\ IBM T. J. Watson Research Center, P.O. Box 218, Yorktown Heights, New York 10598, USA \\ (Received 28 April 2005; revised manuscript received 12 July 2005; published 7 September 2005)
}

\begin{abstract}
In this paper, we combine the modified electrostatics of a one-dimensional transistor structure with a quantum kinetic formulation of Coulomb interaction and nonequilibrium transport. A multi-configurational self-consistent Green's function approach is presented, accounting for fluctuating electron numbers. On this basis we provide a theory for the simulation of electronic transport and quantum charging effects in nanotransistors, such as a gated carbon nanotube and whisker devices and one-dimensional CMOS transistors. Singleelectron charging effects arise naturally as a consequence of the Coulomb repulsion within the channel.
\end{abstract}

DOI: 10.1103/PhysRevB.72.125308 PACS number(s): 73.63. - b, 72.10. - d, 73.23. - b, 73.22. -f

\section{INTRODUCTION}

As scaling of field-effect-transistor (FET) devices reaches the decananometer regime, multigate architectures, and ultrathin active channel regions are mandatory in order to preserve electrostatic integrity. It has been shown that a coaxially gated nanowire represents the ideal device structure for ultimately scaled FETs. ${ }^{1,2}$ A variety of one-dimensional (1D) nanostructures-such as carbon nanotubes, silicon nanowires, or compound semiconductor nanowhiskers-have been demonstrated and intensive research has been devoted to the realization of field-effect-transistor action in these nanostructures. ${ }^{3-6}$ Due to the small lateral extent in the nanometer range, electronic transport through such nanowires is one dimensional with only a few or even a single transverse mode participating in the current. As a result, increasingly less electrons are involved in the switching of a nanowire transistor. In fact, even in devices with a rather long channel lengths of $100 \mathrm{~nm}$, only on the order of 1-10 electrons constitute the charge in the channel for on-state voltage conditions. Hence, single-electron charging effects are increasingly important and have to be taken into account. ${ }^{7-9}$

Two different approaches are commonly used to describe electronic transport in nano-transistors: A quantum kinetic approach based on the real-time Green's functions provides an excellent description of nonequilibrium states. ${ }^{10-12}$ Here, the Coulomb interaction is described in terms of a selfconsistent Hartree potential, optionally combined with a spin-density-functional exchange-correlation term in local density approximation (LDA-SDFT). However, this framework does not account for single-electron charging effects without forcing integer electron numbers. Alternatively, the second approach considers a quasi-isolated nanosystem with a many-body formulation of Coulomb interaction, including electronic transport on a basis of rate equations. ${ }^{13-19}$ While predicting single-electron charging effects correctly, the latter neglects dissipation and renormalization effects due to the source and drain contacts.

Here, we present an approach that allows us to combine a quantum kinetic description of nonequilibrium electron transport with nonlocal many-body Coulomb effects in onedimensional FET nanodevices for application-relevant temperatures. Within our approach, single-electron charging effects arise naturally as a consequence of the Coulomb interaction. Our formalism contains two central ingredients: In order to cope with particle-number fluctuations (i.e., noninteger expectation values) under nonequilibrium conditions, we introduce a multiconfigurational self-consistent Green's function algorithm. Second, we consider a one-dimensional Coulomb Green's function for the transistor channel that allows us to properly incorporate many-body interaction effects into a quantum kinetic approach with electrostatic boundary conditions for a realistic FET. As an example, we calculate the transfer characteristics of a nanowire transistor with Schottky barriers (SB) at the contact-channel interfaces.

\section{COULOMB GREEN'S FUNCTION}

Consider a coaxially gated field-effect-transistor, as illustrated in Fig. 1. A cylindrical semiconducting channel material is surrounded by a thin dielectric and a metallic gate electrode. The electrostatic potential $V$ inside such a onedimensional (1D) transistor channel obeys a modified Poisson equation, 1,20

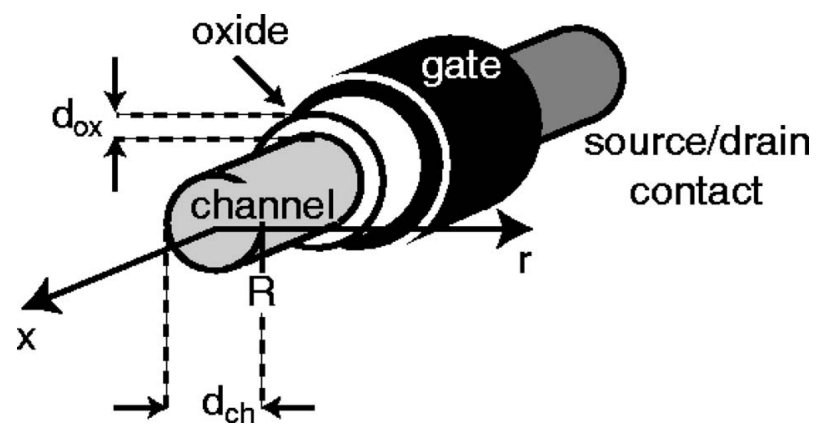

FIG. 1. Schematic view of a 1D FET geometry. $\left(d_{o x}\right.$ and $d_{c h}$ denote the gate insulator thickness and the channel diameter of the nanotransistor, respectively.) 


$$
\frac{\partial^{2}}{\partial x^{2}} V(x)-\frac{1}{\lambda^{2}} V(x)=-\frac{1}{\epsilon_{0} \epsilon_{c h} S} \rho(x)-\frac{1}{\lambda^{2}} V_{G},
$$

where $\rho$ is the 1D charge density. $V_{G}$ denotes the gate potential and $S=\pi d_{c h}^{2} / 4$ is the effective cross-sectional area. The characteristic length $\lambda$ is related to the spatial separation of the gate electrode from the channel (which should be smaller than the total length $L$ of the channel). ${ }^{1,20}$ Note that Eq. (1) is an appropriate description for coaxial as well as planar transistor geometries, differing only in the characteristic length $\lambda$. In the following, we assume perfect metallic source and drain contacts at the boundaries, yielding fixed-potential boundary conditions due to given chemical potentials within these contacts.

A key ingredient in our formalism is the usage of a Coulomb Green's function ${ }^{21}$ for the description of charge interaction within the channel. This allows us to formulate classical electrostatics (with boundary conditions) and the manybody interaction between electrons on equal footing. The corresponding Coulomb Green's function of the gated channel (with $0 \leqslant x, x^{\prime} \leqslant L$ and vanishing potential at the boundaries $0, L)$ can readily be obtained as

$$
\begin{aligned}
v\left(x, x^{\prime}\right)= & \frac{\lambda}{2}\left(e^{-\left|x-x^{\prime}\right| / \lambda}-e^{-\left(x+x^{\prime}\right) / \lambda}\right) \\
& +\frac{\lambda}{2} e^{-L / \lambda}\left(\frac{\cosh \left(\frac{x-x^{\prime}}{\lambda}\right)}{\sinh \left(\frac{L}{\lambda}\right)}-\frac{\cosh \left(\frac{x+x^{\prime}}{\lambda}\right)}{\sinh \left(\frac{L}{\lambda}\right)}\right) .
\end{aligned}
$$

[In contrast, if we considered open boundary conditions, we would obtain $v\left(x, x^{\prime}\right)=(\lambda / 2) \exp \left(-\left|x-x^{\prime}\right| / \lambda\right)$ instead.] For a given charge density $\rho$ inside the channel, the potential thus reads as

$$
V(x)=\frac{1}{\epsilon_{0} \epsilon_{c h} S} \int d x^{\prime} v\left(x, x^{\prime}\right) \rho\left(x^{\prime}\right)+V_{e x t}(x),
$$

with the external potential contribution

$$
\begin{aligned}
V_{\text {ext }}(x)= & \frac{\sinh \left(\frac{L-x}{\lambda}\right)}{\sinh \left(\frac{L}{\lambda}\right)} V_{S}+\frac{\sinh \left(\frac{x}{\lambda}\right)}{\sinh \left(\frac{L}{\lambda}\right)} V_{D} \\
& +\frac{1}{\lambda^{2}} \int d x^{\prime} v\left(x, x^{\prime}\right) V_{G},
\end{aligned}
$$

where $V_{S}$ and $V_{D}$ denote the contact potentials.

\section{SYSTEM HAMILTONIAN}

In this paper, we make use of a tight-binding description of the channel, represented by a localized 1D single-particle basis $\left\{\phi_{j}(x, \sigma)\right\}$ (where the single-particle index $j$ represents a combined orbital, site, and spin multi-index.) The total system Hamiltonian $H=H_{0}+H_{e e}+H_{S}+H_{D}$ can be split into four parts. $H_{0}$ contains all single-particle terms of the transistor channel:

$$
\begin{gathered}
H_{0}=\sum_{j, k} h_{j k} c_{j}^{\dagger} c_{k}, \\
h_{j k}=-e \sum_{\sigma} \int d x \phi_{j}^{*}(x, \sigma) \phi_{k}(x, \sigma)\left[V_{d o p}(x)+V_{e x t}(x)\right] \\
+\delta_{j k} d_{j}+t_{j k},
\end{gathered}
$$

with the electron annihilation operator $c_{j}$ for state $j$. The composition of the channel (material-specific properties, layer sequence, etc.) and possible terms due to image charges in the contacts are described by $d_{j}$ and off-diagonal hopping matrix elements $t_{j k}{ }^{22,23} V_{d o p}$ denotes the potential resulting from fixed charges $\rho_{d o p}$ (due to ionized doping atoms), whereas $V_{\text {ext }}$ stems from external charges due to the applied drain-source voltage and the gate influence [see Eq. (4)].

Furthermore, $H_{S}$ and $H_{D}$ are the Hamiltonians for the source and drain contacts, respectively. The latter also contain the corresponding hopping terms to the outer ends of the channel region, providing electron injection and absorption. Each contact is assumed to be in a state of local equilibrium with an individual chemical potential according to the applied voltage. [See also Eq. (12) below.]

Most importantly, $H_{e e}$ describes the many-body Coulomb interaction between electrons within the channel region, ${ }^{19}$

$$
H_{e e}=\frac{1}{2} \sum_{m, j, k, l} V_{m j k l} c_{m}^{\dagger} c_{j}^{\dagger} c_{k} c_{l}
$$

with Coulomb matrix elements

$$
\begin{aligned}
V_{m j k l}= & \frac{e^{2}}{\epsilon_{0} \epsilon_{c h} S} \sum_{\sigma, \sigma^{\prime}} \int d x \int d x^{\prime} v\left(x, x^{\prime}\right) \\
& \times \phi_{m}^{*}(x, \sigma) \phi_{j}^{*}\left(x^{\prime}, \sigma^{\prime}\right) \phi_{k}\left(x^{\prime}, \sigma^{\prime}\right) \phi_{l}(x, \sigma),
\end{aligned}
$$

which employ the Coulomb Green's function, Eq. (2).

\section{QUANTUM KINETICS}

A quantum kinetic description of the system (under nonequilibrium conditions, in particular) is obtained via the usage of a real-time Green's functions formalism. ${ }^{24-26}$ The retarded and lesser (two-point) Green's functions in the time domain are given by

$$
\begin{gathered}
G_{j k}^{r}(t)=-i \Theta(t)\left\langle\left\{c_{j}(t), c_{k}^{\dagger}(0)\right\}\right\rangle, \\
G_{j k}^{<}(t)=i\left\langle c_{k}^{\dagger}(0) c_{j}(t)\right\rangle,
\end{gathered}
$$

for steady-state conditions. In the following, we will consider the Fourier transformed functions, defined via $G(E)$ $=(1 / \hbar) \int d t \exp (i E t / \hbar) G(t)$.

In matrix notation, the Dyson equation for the channel can be written as ${ }^{10,25-27}$ 


$$
\begin{gathered}
G^{r}=G^{r 0}+G^{r 0} \Sigma^{r} G^{r}, \\
G^{<}=\left(1+G^{r} \Sigma^{r}\right) G^{<0}\left(\Sigma^{a} G^{a}+1\right)+G^{r} \Sigma^{<} G^{a},
\end{gathered}
$$

where $G^{r 0} \equiv(E-h+i \epsilon)^{-1}$ (with $\epsilon \rightarrow 0+$ ) and $G^{a}=G^{r \dagger}$. We assume $G^{<0} \equiv 0$, which means that the channel remains empty without contacts. For application-relevant temperatures $T$ well above the Kondo temperature of the system, the Coulomb interaction can be treated independently of the contact coupling, albeit self-consistently. Within this approximation, the total self-energy $\Sigma=\Sigma_{e e}+\Sigma_{S}+\Sigma_{D}$ can be written as a sum of a Coulomb interaction term $\Sigma_{e e}$ and two contact terms $\Sigma_{S}$, $\Sigma_{D}$ of a noninteracting form.

Once $G^{<}$has been obtained self-consistently from Eq. (9), observables like the electron density $\rho_{e}$ and the current $I_{e}$ (through an arbitrary layer at $x_{0}$ ) can be calculated via

$$
\begin{gathered}
\rho_{e}(x, \sigma)=-e \sum_{j, k} \phi_{j}^{*}(x, \sigma) \phi_{k}(x, \sigma) \hat{\rho}_{j k}, \\
I_{e}=-\frac{e}{\hbar} \sum_{\substack{j, k \\
x_{j} \leqslant x_{0}, x_{k}>x_{0}}} 2 \operatorname{Im}\left(t_{j k} \hat{\rho}_{j k}\right),
\end{gathered}
$$

with the single-particle density matrix

$$
\hat{\rho}_{j k}=\frac{1}{2 \pi} \int d E \frac{1}{i} G_{k j}^{<}(E) .
$$

The effective contact self-energies due to the coupling of the channel to the source and drain regions $(c=S, D)$ can be obtained as ${ }^{10,27,28}$

$$
\sum_{c_{j k}}^{r}(E)=\sum_{p, q \in c} t_{c_{j p}} G_{c_{p q}}^{r 0}(E) t_{c_{q k}}
$$

with the isolated contact Green's function $G_{c}^{r 0}$ and contactchannel hopping terms $t_{c}$. The corresponding lesser selfenergy reads as $\Sigma_{c}^{<}=i f_{c} \Gamma_{c}$, where $\Gamma_{c} \equiv i\left(\Sigma_{c}^{r}-\Sigma_{c}^{a}\right) . f_{S}$ and $f_{D}$ are the local source and drain Fermi distribution functions, respectively, assuming local equilibrium within these reservoirs. (Note that $\Sigma_{c}$ is a source for renormalization and dissipation.)

The evaluation of the Coulomb self-energy requires a suitable approximation scheme due to the infinite Green's function hierarchy (which is a consequence of the twoparticle interaction). As a first-order expansion (Hartree-Fock diagrams), four-point Green's functions can be factorized into linear combinations of products of two-point functions. ${ }^{24,27}$ Using this approximation, the Coulomb selfenergy reads as

$$
\sum_{e e_{m l}}^{r}=\sum_{j, k}\left(V_{m j k l}-V_{j m k l}\right) \hat{\rho}_{j k}
$$

Note that $\Sigma_{e e}^{<}=0$, and $\Sigma_{e e}^{r}$ is nonlocal, Hermitian and energy independent (static) within the considered approximation scheme; compare also with Ref. 27. For convenience, the Hartree potential [the first $V$ term in Eq. (13)],

$$
V_{H}(x)=\frac{1}{\epsilon_{0} \epsilon_{c h} S} \sum_{\sigma^{\prime}} \int d x^{\prime} v\left(x, x^{\prime}\right) \rho_{e}\left(x^{\prime}, \sigma^{\prime}\right),
$$

can be separated from the retarded Coulomb self-energy [compare Eq. (3)], where the electron charge density $\rho_{e}$ is given by Eq. (10). Hence, the total electrostatic potential of the system reads as $V=V_{d o p}+V_{H}+V_{e x t}$.

For integer-number electron-filling conditions, Eq. (13) provides an excellent description of the system for application-relevant temperatures. However, under nonequilibrium conditions, one has to deal with noninteger average filling situations, which are beyond the scope of a first-order (mean-field) self-energy in general. In the following section, we will therefore present a multiconfigurational approach that is able to cope with such particle-number fluctuations (of the quantum statistical ensemble).

\section{MULTICONFIGURATIONAL SELF-CONSISTENT GREEN'S FUNCTION}

A thermodynamic state of the transistor channel with fluctuating electron number can be considered as a weighted mixture of many-body states with integer filling (configurations) of relevant single-particle states. For a given $G^{<}$, "relevant" single-particle states of the channel are defined as eigenstates of the matrix $\hat{\rho}$ [Eq. (11)] that exhibit significant occupation fluctuations and have a sufficiently small dephasing (due to the contact coupling), thus focusing on resonantly trapped (i.e., quasibound) electrons. (Typically, among the eigenstates of $\hat{\rho}$ there are also evanescent states that are remnants of the contact modes, exhibiting strong dissipation.) The corresponding eigenvalues of $\hat{\rho}$ can be interpreted as nonequilibrium occupation numbers. This projection to a relevant single-particle subspace of dimension $N$ reduces the resulting Fock subspace dimension $2^{N}$ significantly, rendering this approach numerically feasible. The main idea behind the following considerations is to treat the interaction of relevant states and their contribution to $\Sigma_{e e}$ in a many-body multi-configurational manner, whereas all remaining states are descibed conventionally by means of their mean-field contributions to $\Sigma_{e e}$ via $\hat{\rho}$ only.

For each configuration $\kappa$, a corresponding single-particle density-matrix $\hat{\rho}_{\kappa}$ can be derived from $\hat{\rho}$. We assume that the Coulomb self-energy approximation, Eq. (13), becomes adequate for each configuration, defining a set of self-energies $\Sigma_{e e}^{r}\left[\hat{\rho}_{\kappa}\right]$ (which need not be self-consistent individually). Then the Green's function can be written as a configuration average:

$$
\bar{G}=\sum_{\kappa} w_{\kappa} G\left[\hat{\rho}_{\kappa}\right]
$$

where $w_{\kappa}$ denotes the weight of configuration $\kappa . G\left[\hat{\rho}_{\kappa}\right]$ is the corresponding Green's function (retarded and lesser) that is obtained by using Eq. (9) with an individual $\Sigma_{e e}^{r}\left[\hat{\rho}_{\kappa}\right]$.

Within the relevant Fock subspace, a projected manybody Hamiltonian matrix can be defined as 


$$
H_{\kappa \lambda}^{M B}=\left\langle\kappa\left|H_{0}+H_{e e}+H_{e e}^{e x t}+H_{r e}^{e x t}\right| \lambda\right\rangle,
$$

where $|\kappa\rangle$ denotes a relevant Fock basis state. $H_{0}$ and $H_{e e}$ are defined in Eqs. (5) and (6), respectively. $H_{e e}^{\text {ext }}$ describes the mean-field interaction of relevant states with the rest (which vanishes in $\left.\left\langle\kappa\left|H_{e e}\right| \lambda\right\rangle\right)$ :

$$
H_{e e}^{e x t}=\sum_{j, k} \Sigma_{e e_{\mathrm{jk}}}^{r}\left[\hat{\rho}_{\kappa=v a c}\right] c_{j}^{\dagger} c_{k},
$$

where $\rho_{\kappa=v a c}$ is the single-particle density matrix for the vacuum configuration within the relevant subspace. Furthermore, $H_{r e}^{\text {ext }}$ contains projected renormalization terms due to the contacts [i.e., the Hermitian part of $\Sigma_{c}^{r}$ of Eq. (12) evaluated at suitable energies]. Note that the configurations defined above might not be exact eigenstates of $H^{M B}$. In the following, we restrict ourselves to the dominant diagonal elements $E_{\kappa}=H_{\kappa \kappa}^{M B}$ and neglect projected dissipation terms.

The weight vector $w$ defines a projected nonequilibrium many-body statistical operator in the relevant Fock basis. Consequently, the resulting many-body lesser Green's function (containing relevant states only) in a Lehmann representation reads as

$$
G_{M B}^{<}[w]_{j k}(t)=i \sum_{\kappa, \lambda} w_{\kappa} e^{(i / \hbar)\left(E_{\lambda}-E_{\kappa}\right) t}\left\langle\kappa\left|c_{k}^{\dagger}\right| \lambda\right\rangle\left\langle\lambda\left|c_{j}\right| \kappa\right\rangle,
$$

where $|\kappa\rangle$ denotes a relevant Fock state with energy $E_{\kappa}$. In principle, $w$ must be chosen such that $G_{M B}^{<}[w]$ resembles the given $G^{<}$for contributions originating from relevant states, i.e., $\Delta\left(G_{M B}^{<}[w], G^{<}\right)=\min$ within the relevant subspace, where $\Delta$ measures the cumulative difference of spectral weights of corresponding resonances. However, for many applications it is sufficient to consider a vector $w$ that maximizes the entropy at an effective temperature $T^{*}\left(T, V_{G}, V_{D}\right)$ under the (weaker) subsidiary condition that $\Sigma_{\kappa} w_{\kappa} \hat{\rho}_{\kappa}=\hat{\rho}$. With this approximation we obtain ${ }^{19,29,30}$

$$
w_{\kappa}=\frac{1}{Z} \exp \left[-\frac{1}{k_{B} T^{*}}\left(E_{\kappa}-\sum_{r} \mu_{r} n_{r}(\kappa)\right)\right],
$$

where $Z$ is a normalization constant. $n_{r}$ denotes the occupation number of the relevant single-particle state $r$, and $\mu_{r}$ represents the individual chemical potential that is a Lagrange parameter for the subsidiary condition above. (Under moderate bias conditions, it is justified to assume $T^{*}$ $\approx T$.)

In turn, $\bar{G}^{<}$from Eq. (15) for this $w$ can be taken as a new $G^{<}$, serving as an input for the calculation scheme described above. This defines a self-consistency procedure for $\bar{G}$ and $w$, which we refer to as the multiconfigurational selfconsistent Green's function algorithm (MCSCG). Such an approach resembles the multiconfigurational self-consistent field (MCSCF) approximation to some extent. ${ }^{31}$ However, MCSCG deals with grand-canonical nonequilibrium states and considers an incoherent superposition (mixture) of different configurations. Obviously, coherent superpositions of many-body states of varying particle numbers would be subject to strong dephasing due to the Coulomb interaction and

\begin{tabular}{|c|c|}
\hline 1. & $\begin{array}{l}\text { Start implementation of function } G^{<}, \\
\text {e.g., via the Hartree-Fock calculation }\end{array}$ \\
\hline 2. & Function $G^{<}$given \\
\hline 3. & $\begin{array}{c}\text { Calculate single-particle density matrix } \hat{\rho}\left[G^{<}\right] \\
\text {via numerical integration over } E\end{array}$ \\
\hline 4. & $\begin{array}{c}\text { Diagonalize } \hat{\rho} \\
\text { and choose relevant single-particle states }\end{array}$ \\
\hline 5. & $\begin{array}{c}\text { Calculate matrices } \hat{\rho}_{\kappa} \text { from } \hat{\rho} \\
\text { for all configurations } \kappa \text { of relevant states }\end{array}$ \\
\hline 6. & $\begin{array}{c}\text { Implement functions } G^{r}\left[\hat{\rho}_{\kappa}\right] \\
\text { by use of the Dyson equation } \\
\text { with Hartree-Fock } \Sigma_{e e}^{r}\left[\hat{\rho}_{\kappa}\right] \\
\text { for all relevant configurations } \kappa\end{array}$ \\
\hline 7. & $\begin{array}{l}\text { Implement functions } G^{<}\left[\hat{\rho}_{\kappa}\right] \\
\text { by use of the Dyson equation with } G^{r}\left[\hat{\rho}_{\kappa}\right] \\
\text { for all relevant configurations } \kappa\end{array}$ \\
\hline 8. & $\begin{array}{c}\text { Calculate configuration weight vector } w \\
\text { by use of the many-body } \\
\text { Hamiltonian } H^{M B} \text { such that } \\
\Delta\left(G_{M B}^{<}[w], G^{<}\right)=\text {min } \\
\text { or } \Sigma_{\kappa} w_{\kappa} \hat{\rho}_{\kappa}=\hat{\rho} \text { with max. entropy }\left(T^{*}\right) \\
\text { within the relevant subspace }\end{array}$ \\
\hline 9. & $\begin{array}{l}\text { New implementation of functions } \\
\qquad \begin{aligned} G^{r} & =\Sigma_{\kappa} w_{\kappa} G^{r}\left[\hat{\rho}_{\kappa}\right] \\
G^{<} & =\Sigma_{\kappa} w_{\kappa} G^{<}\left[\hat{\rho}_{\kappa}\right]\end{aligned}\end{array}$ \\
\hline 10. & Loop back to step 2 until convergence \\
\hline 11. & $\begin{array}{c}\text { Calculate single-particle quantities by } \\
\text { use of } G^{<}, G^{r} \\
\text { via numerical integration over } E\end{array}$ \\
\hline
\end{tabular}
the resulting entanglement with the environment.

Table I visualizes our implementation of the MCSCG pro-
TABLE I. Implementation of the MCSCG algorithm.

cedure. Compared to the standard Hartree self-consistency procedure for the electron density $\rho_{e}$ and the Hartree potential $V_{H}$, we thus have the generalizations $\rho_{e} \rightarrow G^{<},\left\{w_{\kappa}\right\}$ and $V_{H} \rightarrow\left\{\Sigma_{e e}\left[\hat{\rho}_{\kappa}\right]\right\}$. Note that the Poisson equation (2) is solved inherently by considering the Coulomb Green's function in Eqs. (6), (7), and (13).

In future implementations of the MCSCG approach, the many-body calculation for $w$ could be extended to include off-diagonal terms of $H^{M B}$ and dissipation effects for relevant states. From a numerical diagonalization of the projected many-body problem based on $H^{M B}$, it is also possible to calculate higher-order correlation functions (within the relevant subspace). Note that the MCSCG can also be interpreted as a means to construct an effective $\bar{\Sigma}_{e e}$ for Eq. (9).

\section{EXAMPLE: SB-FET}

In the following illustrative example, we consider a oneband nanowire FET with Schottky-barrier injectors, having 
one localized orbital (with two spin directions) per site. Therefore, only Coulomb matrix elements of the form $V_{i j j i}$ are remaining. Furthermore, we assume nearest-neighbor hopping with a real hopping parameter $t=\hbar^{2} /\left(2 m^{*} a^{2}\right)$. We have used the following device parameters: The nominally undoped channel has a diameter of $d_{c h}=4 \mathrm{~nm}$ and a length of $L=20 \mathrm{~nm}$ (implemented as 20 sites with a spacing of $a=1 \mathrm{~nm})$. The channel with $\epsilon_{c h}=15$ is surrounded by a gate oxide with $d_{o x}=10 \mathrm{~nm}$ and $\epsilon_{o x}=3.9$, yielding $\lambda \approx 3.7 \mathrm{~nm}$. We assume an effective electron mass of $m^{*}=0.05 m_{e}$ (giving $t=0.77 \mathrm{eV}$ ). The metallic source and drain contacts have a Schottky-barrier height of $\Phi_{S B}=0.5 \mathrm{eV}$. For simplicity, the corresponding contact self-energy is assumed to be of the form $\Sigma_{c}^{r} \approx-i \Gamma / 2$ (within the band at the outer ends of the channel) with $\Gamma \approx 76 \mathrm{meV}$. Note that this parameter has to be chosen to match the actual metal contact used in an experiment. However, it is uncritical for the electronic spectrum and single-electron charging effects. The system temperature is $T=77 \mathrm{~K}$. The Lagrange parameters for the entropy maximization at a given temperature and individual (nonequilibrium) occupation numbers within the MCSCG were obtained with the help of a Newton iteration scheme. Up to $N=6$ adaptively chosen relevant single-particle states were taken into account, depending on the applied voltages (with $\left.V_{S} \equiv 0\right)$.

Figure 2 visualizes the local density of states (LDOS) for low drain-source bias conditions and two different gate voltages $V_{G}=0.59 \mathrm{~V}$ and $V_{G}=0.71 \mathrm{~V}$, where the average electron number in the channel becomes $N_{e} \approx 0$ and $N_{e} \approx 1$, respectively. The existence of quasibound states (i.e., spatially and energetically localized resonances in the spectral function $A$ ) yields discrete single-electron energies with associated interaction energies due to $\Sigma_{e e}^{r}$. Comparing the situation for $N_{e}=1$ with $N_{e}=0$, the single-electron resonances are moved to higher energies with respect to the lowest-energy state due to the Coulomb repulsion. Note that each electron is not subject to its own Hartree potential (see the lowest resonance in Fig. 2(b)) because $\Sigma_{e e}^{r}$ does not contain unphysical self-interaction energies, but includes exchange terms and correctly accounts for the electron spin. In the shown example, the next higher available state for a second electron (with opposite spin) is separated by the interaction energy $V_{1221} \approx 93 \mathrm{meV}$ (see the arrow in Fig. 2(b)). In general, energy levels are split by exchange energy terms, which have a significant influence on the energy spectrum.

As a natural consequence of $h+\sum_{e e}^{r}$ we therefore expect to observe the effect of a steplike electron filling (under conditions close to equilibrium in particular), energetically determined by single-electron levels and repulsion energies. This behavior in fact can be seen in Fig. 3, where the electronfilling characteristics is plotted for a varying gate voltage and fixed drain-source bias $V_{D}=2 \mathrm{mV}$. Furthermore, Coulomb oscillations in the accompanying current through the channel can be identified. For example, the separation of the first two conductance peaks on the gate voltage axis $\Delta V_{G}(1 \rightarrow 2)$ $\approx 0.12 \mathrm{~V}$ is determined by the Coulomb interaction energy $V_{1221}$, scaled by the reciprocal efficiency of the gate at changing the electrostatic potential in the channel. (Note that the applied gate voltage not only shifts the potential but also modifies the confinement slightly, and hence the quasibound states and their interaction energies.) In turn, one could define an effective capacitance $C_{G}^{*}$ via $\Delta V_{G}(1 \rightarrow 2)=e / C_{G}^{*}$.

Models solely based on a self-consistent Hartree potential do not provide quantization effects due to Coulomb repulsion. With a Hartree approach (as used with conventional Schrödinger-Poisson solvers), spectral features are solely shifted in energy, depending on the average electron density. In contrast, the MCSCG (as well as the exact diagonalization of the isolated system) provides a superposition of fading spectra of integer electron numbers with full interaction energies, however, having spectral weights that depend on the average filling condition. The local density of states under
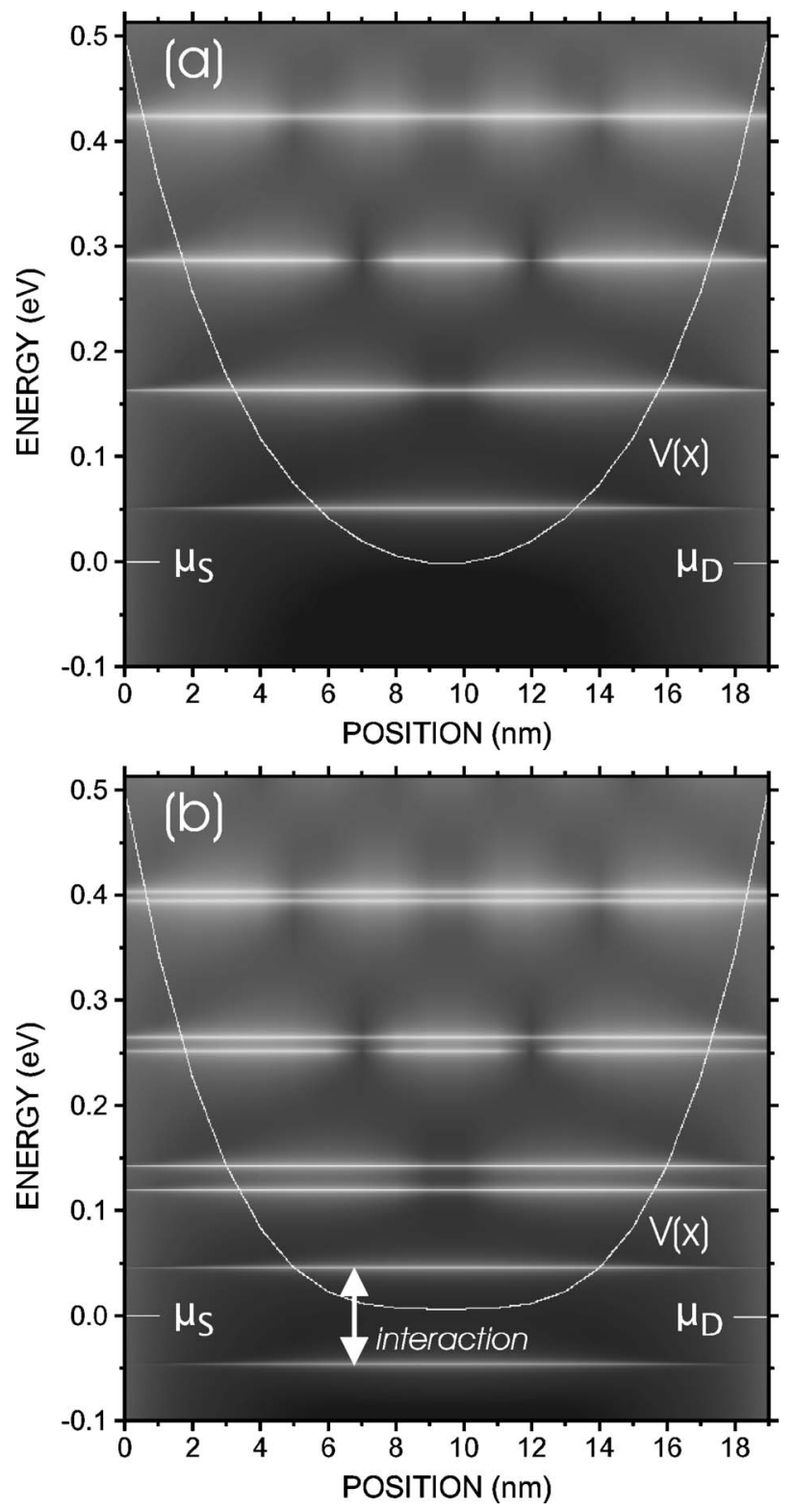

FIG. 2. Spectral function $A(x, E)$ as a gray scale plot for (a) $V_{G}=0.59 \mathrm{~V}$ and (b) $V_{G}=0.71 \mathrm{~V}$. In both cases, $V_{D}=2 \mathrm{mV}$ is chosen. The resulting average electron number within the channel is (a) $N_{e} \approx 0$ and (b) $N_{e} \approx 1$ (the electron is located in the lowest resonance). The solid white line represents the mean-field potential $V(x)$, whereas $\mu_{S}$ and $\mu_{D}$ denote the chemical potentials of the source and drain contact, respectively. $T=77 \mathrm{~K}$. 


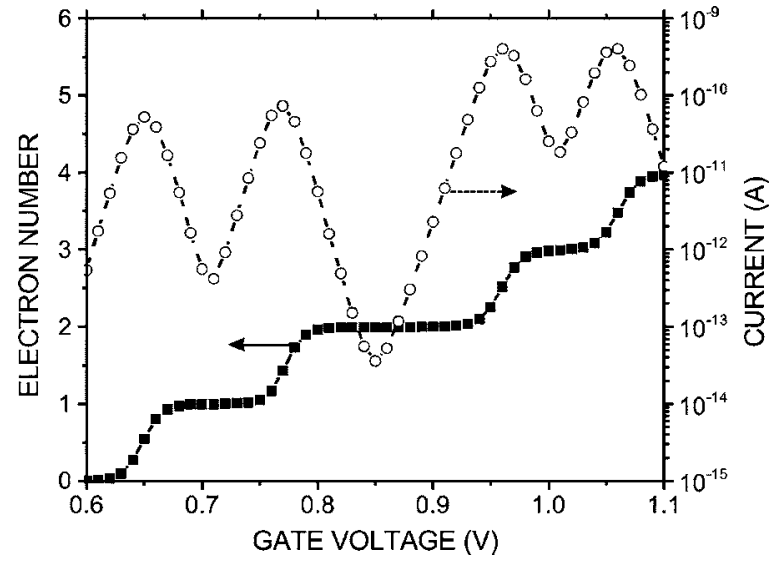

FIG. 3. Single-electron tunneling characteristics for $V_{D}=2 \mathrm{mV}$. The solid line with filled squares corresponds to the average electron number within the potential well, whereas the dashed line with open circles shows the drain current, exhibiting Coulomb oscillations. $T=77 \mathrm{~K}$.

nonequilibrium conditions as shown in Fig. 4 clearly demonstrates this behavior, where the average electron number within the well is $N_{e} \approx 0.22$. In fact, the expectation value of the electron number need not be an integer, especially under nonequilibrium bias conditions, which can be seen in the corresponding transfer characteristics of the system as plotted in Fig. 5. Furthermore, Fig. 6 visualizes the output IV characteristics, where a finite drain voltage is required to pull the chemical potential of the drain contact below the lowestenergy level. The resulting saturation in the drain current with increasing drain voltage is due to the single-mode transport, limited by the source barrier, which is hardly influenced by the drain contact (for the discussed channel length $L \gg \lambda$ ).

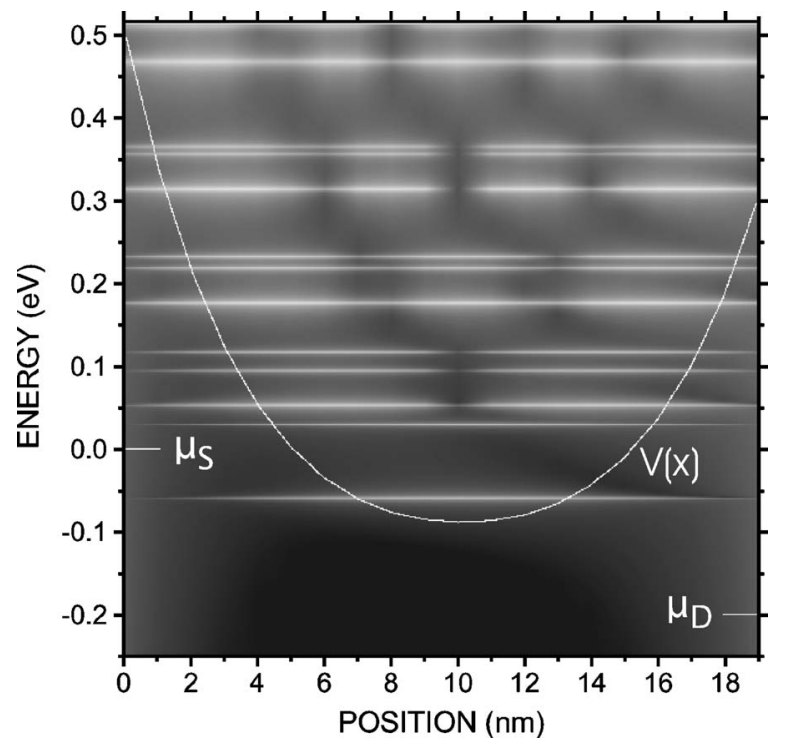

FIG. 4. Nonequilibrium spectral function $A(x, E)$ as a gray scale plot for $V_{G}=0.7 \mathrm{~V}$ and $V_{D}=0.2 \mathrm{~V}$. The resulting average electron number within the channel is $N_{e} \approx 0.22$. [The solid white line represents the mean-field potential $V(x)$, whereas $\mu_{S}$ and $\mu_{D}$ denote the chemical potentials of the source and drain contact, respectively.] $T=77 \mathrm{~K}$.

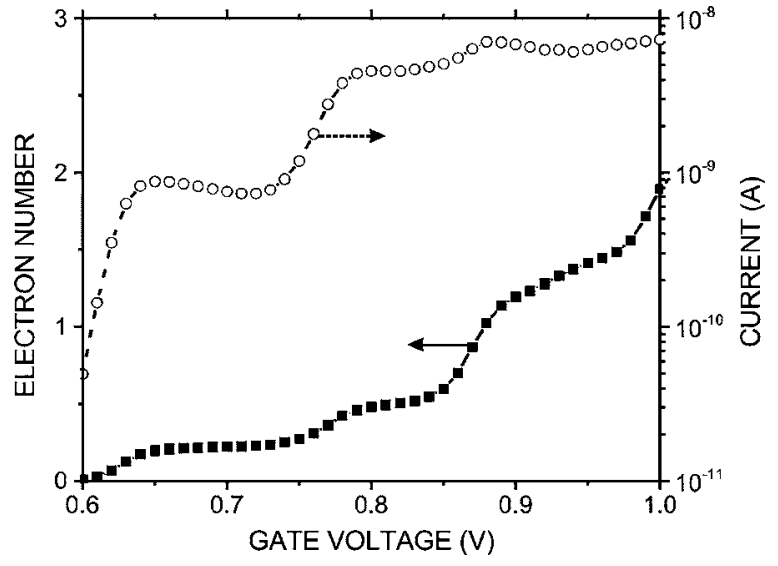

FIG. 5. Transfer characteristics for $V_{D}=0.2 \mathrm{~V}$. The solid line with filled squares corresponds to the average electron number within the potential well, whereas the dashed line with open circles shows the drain current through the channel. $T=77 \mathrm{~K}$.

These results clearly demonstrate the strengths of the MCSCG approach: Compared to a conventional nonequilibrium Green's function approach with a mean-field interaction, the MCSCG provides an advanced description of fluctuating electron numbers (i.e., noninteger filling) of resonantly trapped electrons and hence single-electron charging effects. On the other hand, compared to a rate-equation approach, the MCSCG not only contains a nonequilibrium many-body description of resonantly trapped electrons but also incorporates nonresonant states and their nonequilibrium charging in a consistent manner.

In general, we expect the many-body Coulomb interaction to have a significant impact on the electrical behavior of nanotransistors if the single-electron charging energy becomes $\geqslant 4 k T$, having consequences for the transconductance, onset/pinch-off voltages, subthreshold currents, and system capacitance. A more detailed discussion of these aspects will be published elsewhere.

\section{CONCLUSION}

The Coulomb Green's function of a one-dimensional FET in combination with a quantum kinetic description of elec-

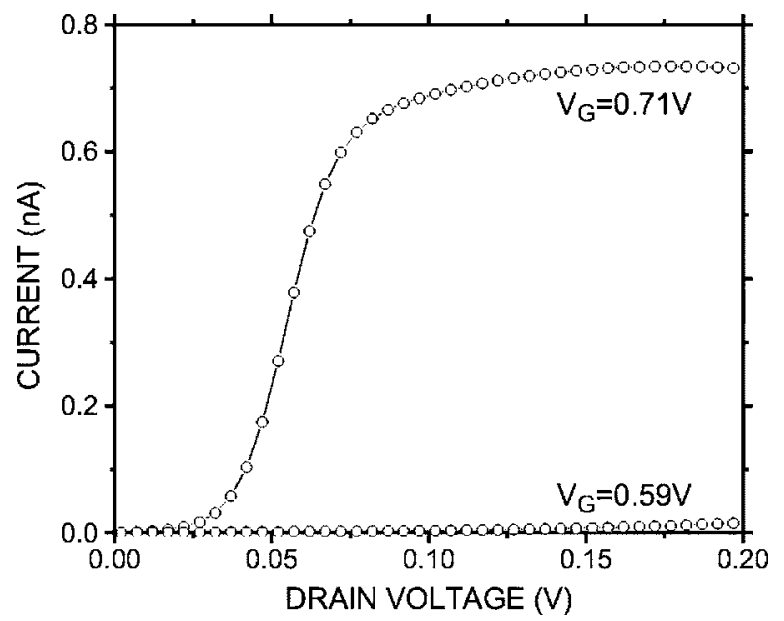

FIG. 6. Output characteristics for $V_{G}=0.59 \mathrm{~V}$ and $V_{G}=0.71 \mathrm{~V}$. $T=77 \mathrm{~K}$. 
tronic transport enables us to describe many-body charging effects within the transistor channel for application-relevant temperatures. We have presented a multiconfigurational selfconsistent Green's function algorithm, which is able to cope with fluctuating electron numbers under nonequilibrium conditions. In the discussed example of a nano-FET with Schottky-barrier injectors, we have visualized how singleelectron charging effects arise naturally as a consequence of the many-body Coulomb repulsion between quasibound states. The usage of a Green's function formulation permits the systematic extension to further Coulomb diagrams and the consistent inclusion of phonon scattering.

With the presented theoretical approach, we are able to describe electronic transport and quantum charging effects in 1D nanotransistors such as gated carbon nanotubes, semiconductor whiskers, and 1D CMOS transistors (in coaxial and multigate planar geometry).
*Electronic address: m.indlekofer@fz-juelich.de

${ }^{1}$ C. P. Auth and J. D. Plummer, IEEE Electron Device Lett. 18, 74 (1997).

${ }^{2}$ H.-S. P. Wong, IBM J. Res. Dev. 46, 133 (2002).

${ }^{3}$ A. Javey, J. Guo, M. Paulsson, Q. Wang, D. Mann, M. Lundstrom, and H. Dai, Phys. Rev. Lett. 92, 106804 (2004).

${ }^{4}$ Y.-M. Lin, J. Appenzeller, J. Knoch, and P. Avouris, cond-mat/ 0501690 (2005), accepted for publication in IEEE Trans. Nanotechnol.

${ }^{5}$ M. C. McAlpine, R. S. Fiedman, S. Jin, K.-H. Lin, W. U. Wang, and C. M. Lieber, Nano Lett. 3, 1531 (2003).

${ }^{6}$ C. Thelander, T. Martensson, M. T. Bjoerk, B. J. Ohlson, M. W. Larsson, L. R. Wallenberg, and L. Samuelson, Appl. Phys. Lett. 83, 2052 (2003).

${ }^{7}$ N. Yoneya, E. Watanabe, K. Tsukagoshi, and Y. Aoyagi, Appl. Phys. Lett. 79, 1465 (2001).

${ }^{8}$ M. Suzuki, K. Ishibashi, K. Toratani, D. Tsuya, and Y. Aoyagi, Appl. Phys. Lett. 81, 2273 (2002).

${ }^{9}$ I. Amlani, R. Zhang, J. Tresek, and R. K. Tsui, J. Vac. Sci. Technol. B 21, 2848 (2003).

${ }^{10}$ R. Lake, G. Klimeck, R. C. Bowen, and D. Jovanovic, J. Appl. Phys. 81, 7845 (1997).

${ }^{11}$ X. Yongqiang, S. Datta, and M. A. Ratner, J. Chem. Phys. 281, 151 (2002).

${ }^{12}$ X. Yongqiang, S. Datta, and M. A. Ratner, J. Comput. Phys. 115, 4292 (2001).

${ }^{13}$ C. W. J. Beenakker, Phys. Rev. B 44, 1646 (1991).

${ }^{14}$ D. V. Averin, A. N. Korotkov, and K. K. Likharev, Phys. Rev. B 44, 6199 (1991).
${ }^{15}$ D. Jovanovic and J.-P. Leburton, Phys. Rev. B 49, 7474 (1993).

${ }^{16}$ D. Weimann, W. Häusler, and B. Kramer, Phys. Rev. Lett. 74, 984 (1995).

${ }^{17}$ Y. Tanaka and H. Akera, Phys. Rev. B 53, 3901 (1996).

${ }^{18}$ D. Pfannkuche and S. E. Ulloa, Phys. Rev. Lett. 74, 1194 (1995).

${ }^{19}$ K. M. Indlekofer and H. Lüth, Phys. Rev. B 62, 13016 (2000).

${ }^{20}$ F. G. Pikus and K. K. Likharev, Appl. Phys. Lett. 71, 3661 (1997).

${ }^{21}$ L. D. Hallam, J. Weis, and P. A. Maksym, Phys. Rev. B 53, 1452 (1996).

${ }^{22}$ P. Vogl, H. P. Hjalmarson, and J. D. Dow, J. Phys. Chem. Solids 44, 365 (1983).

${ }^{23}$ J. A. Støvneng and P. Lipavský, Phys. Rev. B 49, 16494 (1994).

${ }^{24}$ W. Schäfer and M. Wegener, Semiconductor Optics and Transport Phenomena (Springer-Verlag, 2002).

${ }^{25} \mathrm{H}$. Haug and A.-P. Jauho, Quantum Kinetics in Transport and Optics of Semiconductors (Springer-Verlag, Berlin, 1998).

${ }^{26}$ S. Datta, Electronic Transport in Mesoscopic Systems (Cambridge University Press, Cambridge, 1995).

${ }^{27}$ L. E. Henrickson, A. J. Glick, G. W. Bryant, and D. F. Barbe, Phys. Rev. B 50, 4482 (1994).

${ }^{28}$ K. M. Indlekofer, J. Lange, A. Förster, and H. Lüth, Phys. Rev. B 53, 7392 (1996).

${ }^{29}$ K. M. Indlekofer, J. P. Bird, R. Akis, D. K. Ferry, and S. M. Goodnick, Appl. Phys. Lett. 81, 2861 (2002).

${ }^{30}$ K. M. Indlekofer, J. P. Bird, R. Akis, D. K. Ferry, and S. M. Goodnick, J. Phys.: Condens. Matter 15, 147 (2003).

${ }^{31}$ M. W. Schmidt and M. S. Gordon, Annu. Rev. Phys. Chem. 49, 233 (1998). 